\title{
Current recommendations for the treatment of mild asthma
}

This article was published in the following Dove Press journal:

Journal of Asthma and Allergy

8 December 2010

Number of times this article has been viewed

\section{Neal Shahidi \\ J Mark FitzGerald}

Division of Respiratory Medicine, Faculty of Medicine, University of British Columbia, Vancouver, British Columbia, Canada
Correspondence: J Mark FitzGerald The Lung Centre, Vancouver General Hospital, 2775 Laurel St, Vancouver, BC V5Z IM9, Canada

Tel +I 6048754122

$\mathrm{Fax}+\mathrm{I} 6048754695$

Email markf@interchange.ubc.ca
Abstract: Patients suffering from mild asthma are divided into intermittent or persistent classes based on frequency of symptoms and reliever medication usage. Although these terms are used as descriptors, it is important to recognize the approach of focusing on asthma control in managing asthma patients. Beta-agonists are considered first-line therapy for intermittent asthmatics. If frequent use of beta-agonists occurs more than twice a week, controller therapy should be considered. For persistent asthma, low-dose inhaled corticosteroids are recommended in addition to reliever medication. Compliance to regular therapy can pose problems for disease management, and while intermittent controller therapy regimens have been shown to be effective, it is imperative to stress the value of regular therapy especially if an exacerbation occurs. It is also important when such an approach is adopted that there is regular re-evaluations of asthma control. This is because regular anti-inflammatory therapy may become necessary if symptoms become more persistent. Other therapies are seldom needed. Antileukotrienes can be considered an option for mild asthma; however, studies have shown that they are not as effective as inhaled corticosteroids. Aside from therapy, patient education, which includes a written action plan, should be a component of the patient's strategy for disease management.

Keywords: mild asthma, treatment, inhaled corticosteroids, asthma education

\section{Introduction}

Mild asthma patients make up by far the biggest number of asthma patients. In a recent study of the economic burden of asthma among a population-based cohort of over 150,000 patients, $67.1 \%$ were mild, $25.5 \%$ were moderate, and $7.4 \%$ were classified as severe. The severe group was responsible for over $60 \%$ of the health care costs, ${ }^{1}$ but interestingly there was a significant burden in a minority of patients who were deemed to be mild at baseline but subsequently became poorly controlled. Mild intermittent asthma is defined as having symptoms less than once a week, and having forced expiratory volume in one second $\left(\mathrm{FEV}_{1}\right)$ or peak expiratory flow $(\mathrm{PEF}) \geq 80 \%$ predicted with $<20 \%$ variability. Mild persistent asthma differs in that symptoms frequency is greater than once per week but less than once per day, and variability in $\mathrm{FEV}_{1}$ or PEF is $<20 \%-30 \%{ }^{2}$

Patients are generally not troubled by nocturnal awakenings and have a reasonable level of daily activity, especially in intermittent asthmatics. The concept of current control and future risk is important with all levels of asthma control but especially in patients with mild asthma. Patients may be willing to accept mild symptoms and have poor adherence with preventive therapy, not recognizing that these treatments are designed not only to control current asthma but also reduce the future risk of asthma exacerbations. 
Most notably in a retrospective study of asthma deaths in Australia, among children and adolescents, over one-third of asthma patients who died from an acute asthma attack had been categorized as having mild asthma by either their parents or primary care physicians. ${ }^{3}$ In this review we focus on the management of mild asthma and base our recommendations primarily on the results of systematic reviews of the literature. Where appropriate, key individual studies will be cited. The review will also primarily focus on adult asthma.

\section{Mild intermittent asthma}

Beta 2-agonists

Inhaled short-acting beta 2-agonists (SABAs) provide rapid relief by reducing airway narrowing and its associated symptoms (cough, chest tightness, wheezing) via relaxation of smooth muscle within the airway walls. SABA therapy works within 5 minutes and relieves asthma symptoms for 3-6 hours. However, SABAs do not control the inflammatory process associated with asthma. Current guidelines

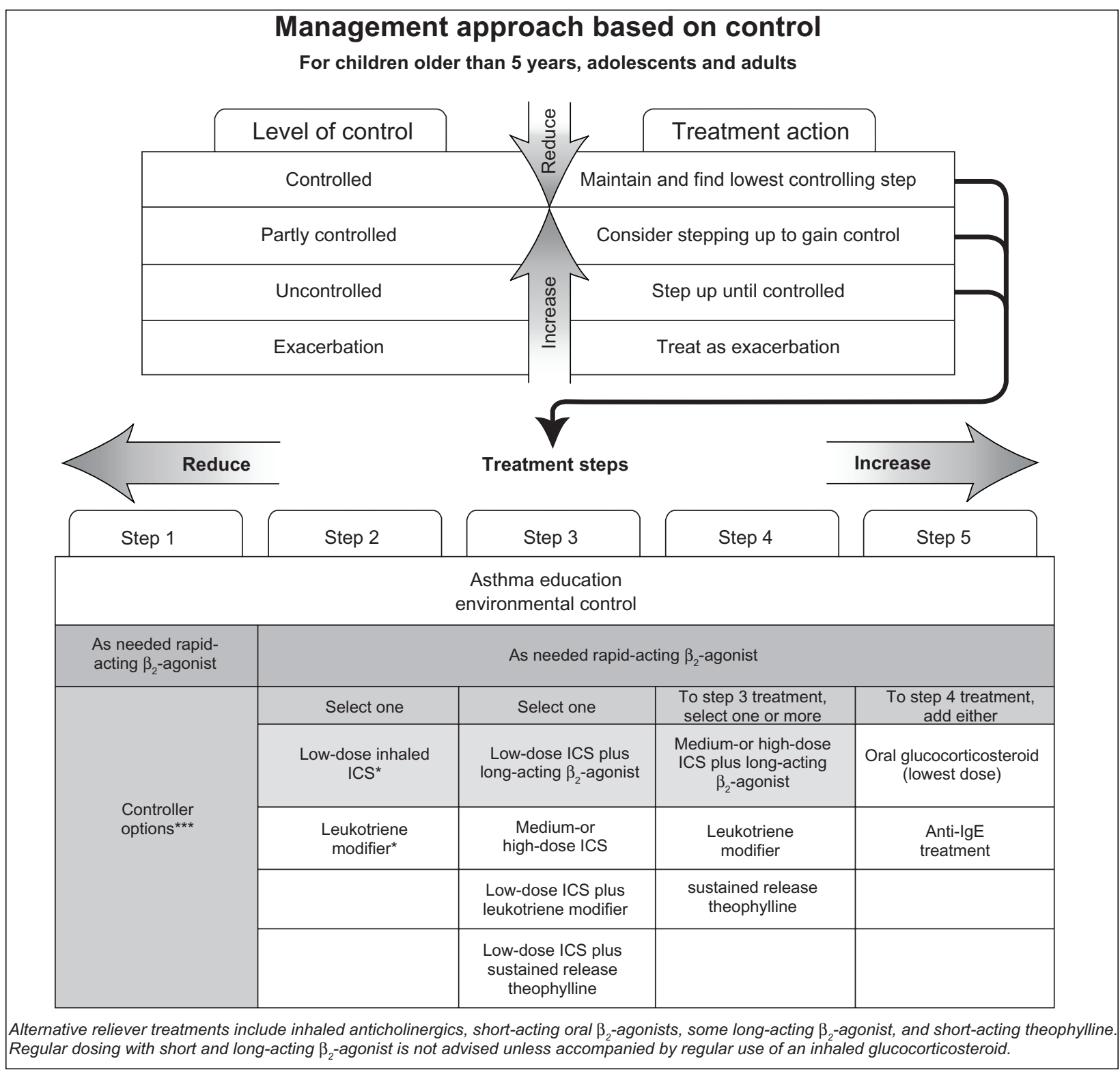

Figure I Management protocol for asthma. Copyright @ 2009, European Respiratory Society. Adapted with permission from National Institutes of Health, Global Initiative For Asthma. GINA Report, Global Strategy for Asthma Management and Prevention. 2009. Available from: http://www.ginasthma.com/Guidelineitem.asp??II=2\&I2=|\&intld=|56।. Accessed 2010 Oct 20.

Notes: "ICS, inhaled glucocorticosteroids; ${ }^{* *}$ Receptor antagonist or synthesis inhibitors; ${ }^{* * * *}$ Preferred controller options are shown in shaded boxes. 
recommend SABAs as the therapy of choice for mild intermittent asthma (see Figure 1). ${ }^{2,4}$ SABA usage should be limited to an as-needed basis, at the lowest dosage and frequency allowing for symptomatic relief. ${ }^{5}$ As-needed therapy is preferred to continuous therapy due to the infrequent nature of symptoms in intermittent mild asthma as well as the lack of benefit and potential risks from sustained therapy especially in the absence of concomitant anti-inflammatory therapy. ${ }^{6}$ Patients can also use the bronchodilator for symptom relief and every 4-6 hours for up to 24 hours in the presence of a viral respiratory infection. Furthermore, with as-needed therapy, the frequency of SABA usage can be used as a measure of asthma control. Once symptom relief with rescue medication is required more than twice weekly there should be early consideration for the introduction of antiinflammatory therapy. A useful analogy for patients taking only reliever therapy is to the use of analgesia for a dental cavity: the pain is relieved but the cavity remains. Similarly, use of a symptom reliever as regular treatment has the potential to mask the inflammatory changes that are associated with even mild asthma.

\section{Inhaled corticosteroids}

When reliever therapy is required more than twice weekly, anti-inflammatory therapy should be introduced. This recommendation is primarily based on evidence indicating clinical benefit with no significant risk from the long-term use of inhaled corticosteroids (ICSs) for asthma treatment. ICSs are also recommended based on evidence that even in mild asthma there is a significant airway inflammatory component. ${ }^{7}$

In a recent study, 96 mild intermittent asthma patients were randomly allocated to either treatment with SABA monotherapy or in addition with low dose ICS (beclomethasone diproprionate $250 \mu \mathrm{g} /$ day). ${ }^{8}$ After 6 months, the SABA and ICS therapy showed a statistically significant improvement in $\mathrm{FEV}_{1}(3.58 \mathrm{~L}$ versus $3.66 \mathrm{~L})$ as well as improvements in FVC, $\mathrm{FEV}_{1} / \mathrm{FVC}$, and PEF. In comparison, SABA monotherapy showed a statistically significant decrease in all analyzed spirometric parameters. However, there was no statistically significant variation between monotherapy and ICS combined with as-needed SABA with respect to lung function measurements. Daily asthma symptom scores significantly decreased ( 0.63 versus 0.30$)$ with ICS and SABA therapy and were significantly increased (from 0.46 to 0.62 ) in patients taking a SABA only. Lastly, a significant reduction in rescue medication usage was associated with a combination of ICS and as-needed SABA therapy, compared with a significant increase in rescue medication usage associated with SABA monotherapy. The authors concluded that the addition of ICS therapy with as-needed beta 2-agonist was effective in mild intermittent asthma. However, the authors noted limitations including the short duration of the study and the low number of study participants.

\section{Mild persistent asthma ICS therapy}

ICSs are the most important therapy for asthma, including mild intermittent asthma, due to their ability to control airway inflammation (see Table 1). The efficacy of ICS therapy includes reducing asthma symptoms, improving lung function, decreasing the frequency and severity of exacerbations, as well as improving quality of life. These findings have been highlighted in numerous studies as well as Cochrane reviews. ${ }^{9-13}$ In a 2008 Cochrane review, incorporating 86 studies with 16,160 participants, the safety and efficacy of fluticasone was assessed in comparison to placebo. ${ }^{11}$ Twenty-four studies included patients with mild asthma and 19 had patients with mild to moderate asthma. In patients suffering from mild to moderate asthma, who were not on oral steroid therapy, fluticasone was shown to improve symptom scores, $\mathrm{FEV}_{1}$, and morning PEF.

In one of the largest studies specifically focusing on mild persistent asthma, the inhaled steroid treatment as regular therapy in early asthma (START) trial, included approximately 7200 patients (5-66 years) who were randomly assigned to either $400 \mu \mathrm{g}$ budesonide (or $200 \mu \mathrm{g}$ for patients $<11$ years of age) or placebo to assess whether early ICS therapy reduces the risk of severe exacerbations over a 3-year period. ${ }^{13}$ A total of $198 / 3568$ patients within the placebo arm and $117 / 3597$ patients within the budesonide arm (hazard ratio $0.56 ; 95 \%$ confidence interval [CI]: $0.45-0.71$; $P<0.0001)$ experienced at least one severe asthma exacerbation. Moreover, patients receiving budesonide presented with more symptom-free days, and they required fewer

Table I Estimated adult daily doses of inhaled glucocorticoids

\begin{tabular}{llll}
\hline Drug & $\begin{array}{l}\text { Low daily } \\
\text { dose }(\mu \mathrm{g})\end{array}$ & $\begin{array}{l}\text { Medium daily } \\
\text { dose }(\mu \mathrm{g})\end{array}$ & $\begin{array}{l}\text { High daily } \\
\text { dose }(\mu \mathrm{g})\end{array}$ \\
\hline $\begin{array}{l}\text { Beclomethasone } \\
\text { dipropionate }\end{array}$ & $200-500$ & $>500-1000$ & $>1000-2000$ \\
$\begin{array}{l}\text { Budesonide } \\
\text { Ciclesonide }\end{array}$ & $200-400$ & $>400-800$ & $>800-1600$ \\
Flunisolide & $80-160$ & $>160-320$ & $>320-1280$ \\
Fluticasone propionate & $100-250$ & $>250-500$ & $>500-1000$ \\
Mometasone furoate & $200-400$ & $>400-800$ & $>800-1200$ \\
Triamcinolone acetonide & $400-1000$ & $>1000-2000$ & $>2000$ \\
\hline
\end{tabular}

Note: For the appropriate use of inhaled corticosteroid therapy in mild asthma see text. Copyright (C) 2009, European Respiratory Society. Adapted with permission from Bateman ED, Hurd SS, Barnes PJ, et al. Global strategy for asthma management and prevention: GINA executive summary. Eur Respir J. 2008;31:143-178. ${ }^{2}$ 
courses of systemic corticosteroids compared with patients in the placebo arm. With respect to lung function, budesonide significantly increased pre- and post- bronchodilator $\mathrm{FEV}_{1}$ from baseline after 1 year and 3 years compared with placebo. However, although the effect remained significant, the impact of budesonide on pre- and post- bronchodilator FEV decreased over time and was small at 3 years. It was suggested to possibly be due to the fact that placebo participants were more likely to utilize additional therapy.

In 2008, data from the 2-year open-label treatment follow-up study were published from the START trial. ${ }^{14} \mathrm{~A}$ total of 5146 participants ( 2604 budesonide group, 2542 placebo group) were treated with once daily budesonide as continuation of therapy or as add-on therapy from the 3-year doubleblind phase alongside their usual asthma medications. Across the 5-year study period, pre-bronchodilator percent predicted $\mathrm{FEV}_{1}$ increased on average by $3.24 \%$, whereas post-bronchodilator percent predicted $\mathrm{FEV}_{1}$ decreased on average by $2.22 \%$, regardless of randomized treatment. However, there was a significant treatment difference in pre-bronchodilator percent predicted $\mathrm{FEV}_{1}$ $(1.21 \%)$ and post-bronchodilator percent predicted $\mathrm{FEV}_{1}$ $(0.85 \%)$ in favor of budesonide in adults (aged 18 years and older). Nevertheless, at the end of the 2-year open label follow up period, no significant differences were observed between the budesonide and placebo group in terms of preand post-bronchodilator percent predicted $\mathrm{FEV}_{1}$, which were observed during the 3-year double-blind phase, due to rapid catch-up in lung function in the reference group. In secondary outcomes, patients who initially began with budesonide showed a significant decrease in risk for severe asthmarelated events, as well as a decrease in need for additional asthma medication compared with the reference group.

With regard to the safety of ICS therapy, it is important to be aware that local side effects such as oropharyngeal candidiasis, occasional cough, and dysphonia may occur. However, it is very unusual in the absence of high-dose ICS for systemic side effects such as easy bruising, adrenal suppression, cataracts, glaucoma, and decreased bone mineral density to be present. ${ }^{5}$ To alleviate the burden of local side effects, spacer devices, mouth washing, and prodrugs such as ciclesonide can be used. Both male and female adult patients should consider daily calcium and vitamin D supplementation specifically if they are at high risk for osteoporosis or already have osteopenia. As for systemic side effects in adult asthma patients, current evidence implies that at doses of $\leq 400 \mu \mathrm{g}$ /day of budesonide or equivalent systemic adverse effects are not a relevant concern. ${ }^{15,16}$

\section{Regular versus intermittent ICS}

While current guidelines recommend the daily use of low-dose ICS therapy, compliance with regular ICS therapy in mild asthma is of great concern. The possibility of patients using their controller medication intermittently is high. In a recent double-blind trial including 225 adults (the Improving Asthma Control Trial [IMPACT]), the efficacy of intermittent shortcourse ICS therapy either alone or in conjunction with daily ICS or leukotriene receptor antagonist therapy was analyzed. ${ }^{17}$ Within the 1-year study period, variation in morning PEF did not significantly differ between groups. Furthermore, nonsignificant differences between groups were identified in postbronchodilator $\mathrm{FEV}_{1}$ or patients suffering from one or more exacerbations. Asthma quality of life scores did not vary significantly between groups. However, daily in addition to intermittent ICS were significantly superior with respect to pre-bronchodilator $\mathrm{FEV}_{1}$, asthma control scores, number of symptom free days, median sputum eosinophils, and median exhaled nitric oxide. Nevertheless, the authors concluded that it may be feasible to treat with intermittent ICS therapy together with an action plan for patients suffering from mild asthma. An important consideration in making a recommendation for these patients relates to the risk of exacerbations. In the event of an asthma exacerbation, the importance of subsequent regular anti-inflammatory therapy should be emphasized. The impact of asthma exacerbations on decline in $\mathrm{FEV}_{1}$ has been documented. ${ }^{18}$ In addition, if a patient elects not to take regular anti-inflammatory therapy it is important to have intermittent reassessment of the patient's level of asthma control including lung function. It is not unusual in clinical practice to see patients who report intermittent episodes of what are described as "wheezy bronchitis" but on closer questioning are in fact mild asthma exacerbations, and these patients invariably display a loss in lung function. An important consideration with all asthma patients is the need to reassure them about the safety of ICSs. ICSs are safe in the doses usually required for most asthma patients but this is especially true in mild asthma where the dose of ICSs required to achieve asthma control is such that it will not be associated with any systemic risks.

\section{Combination therapy}

Although current guidelines recommend the addition of long-acting beta-2-agonists (LABA) to ICS when control with ICS therapy alone cannot be achieved, ${ }^{4,19}$ the introduction of LABAs without prior ICS therapy is still prevalent. ${ }^{20}$ In a 2009 Cochrane review, 27 trials including 8050 participants with mild to moderate asthma (12 trials with a mean 
baseline $\mathrm{FEV}_{1} \geq 80 \%$ predicted) evaluated the efficacy of a LABA+ICS combination therapy compared to ICS therapy alone in steroid-naïve persistent asthma patients. ${ }^{21}$ When comparing LABA+ICS versus a similar dose of ICS, combination therapy did not significantly lower the risk of exacerbations requiring oral steroids, or exacerbations requiring hospital admissions compared with ICS therapy alone. Moreover, no significant differences were identified in serious adverse events or study withdrawals. However, combination therapy did lead to significant improvement in $\mathrm{FEV}_{1}$ from baseline, reduction in rescue beta-2-agonist usage, and improved symptom control. When specifically analyzing patients with an $\mathrm{FEV}_{1} \geq 80 \%$ predicted, similar results were found with nonsignificant differences in the number of patients with exacerbations requiring systemic steroids (relative risk [RR] 1.02; 95\% CI: 0.67-1.56), number of patients with exacerbations requiring hospitalization (RR $0.21 ; 95 \%$ CI: 0.01-4.27), serious adverse events (RR 1.54; 95\% CI: 0.53-4.45), total withdrawals (RR 0.84; 95\% CI: 0.60-1.17), number of patients withdrawing due to poor asthma control or exacerbation (RR 1.41; 95\% CI: 0.45-4.42), and number of patients withdrawing due to adverse effects (RR 1.39; 95\% CI: 0.55-3.51). Similarly, there was a significant difference in $\mathrm{FEV}_{1}$ at endpoint (mean difference 1.75; 95\% CI: 0.20-3.29) and in morning PEF (mean difference 15.32; 95\% CI: 9.63-21.00) in favor of combination therapy compared with ICS therapy alone.

When comparing LABA+ICS versus a higher dose of ICS therapy, combination therapy was associated with patients being more likely to require oral corticosteroids as well as study withdrawal. Similar results were found during subanalysis for patients with $\mathrm{FEV}_{1}>80 \%$ predicted. No significant difference was identified between groups for risk for serious adverse events both overall and with subanalysis for patients with $\mathrm{FEV}_{1}>80 \%$ predicted. Ultimately, the authors found that combining ICS and LABA therapy did not significantly affect the risk of patients requiring oral corticosteroids or hospitalization in steroid-naïve patients suffering from mild to moderate asthma. Moreover, combination therapy in comparison with increasing ICS dosage appears to be inferior in reducing the risk of exacerbations requiring oral corticosteroids and withdrawals.

In 2007, a 6-month double-blind randomized trial (the Beclomethasone and Salbutamol Treatment [BEST] Study) was undertaken to assess the effectiveness of as-needed single-inhaler beclomethasone and salbutamol (albuterol) versus as-needed salbutamol, daily ICS with as-needed salbutamol, or daily ICS/salbutamol with as-needed salbutamol. ${ }^{22} \mathrm{~A}$ total of 455 patients were included in the study (122 as-needed combination therapy, 118 as-needed salbutamol therapy, 106 regular ICS therapy, 109 regular combination therapy). Significantly higher morning PEF values were observed in the as-needed combination therapy and regular ICS therapy groups compared with as-needed salbumatol, whereas no significant variation was detected between regular ICS therapy and as-needed combination therapy. When directly compared with as-needed salbutamol, regular ICS therapy showed significantly less rescue medication usage. Alternatively, as-needed combination therapy had significantly fewer nocturnal awakenings when compared with as-needed salbutamol therapy. The percentage of patients with at least one exacerbation was significantly lower in both regular ICS therapy (5.66\%), and in as-needed combination therapy (4.92\%) compared with as-needed salbutamol therapy (17.80\%). The authors concluded that as-needed combination therapy has comparative effectiveness to regular ICS therapy in mild asthma.

In a further Cochrane review, including 27 studies ( 25 adult trials) with patients suffering from mild to moderate asthma, the effectiveness and safety of combination therapy of ICS and antileukotrienes was analyzed versus ICS monotherapy. ${ }^{23}$ The addition of antileukotrienes to ICS therapy did not significantly reduce the risk of exacerbations requiring systemic steroids (RR 0.64; 95\% CI: 0.38-1.07) compared with ICS therapy alone. Furthermore, the addition of antileukotriene versus ICS therapy did not significantly impact change in $\mathrm{FEV}_{1}$ (weighted mean difference [WMD] 0.06L; 95\% CI: $-0.01-$ 0.14 ), quality of life (WMD 0.08 ; 95\% CI: $-0.03-0.20$ ), nocturnal awakenings (WMD $-6.25 ; 95 \% \mathrm{CI}$ : $12.72-0.23$ ), or symptom score $(-0.10 ; 95 \%$ CI: $-0.24-0.03)$. Significant differences between groups were identified in change from baseline in morning PEF (WMD $7.65 \mathrm{~L} / \mathrm{min}$; 95\% CI: 3.55 11.75), rescue inhaler use (WMD $-1 ; 95 \%$ CI: -0.5 to -2 puffs/week), and eosinophil counts in favor of antileukotrienes with ICS therapy. Concerning safety, the analysis of risk of overall withdrawals as well as overall adverse effects showed no significant difference between groups.

\section{Antileukotrienes}

While ICSs remain the cornerstone for the treatment of mild asthma, some patients have concerns about the perceived adverse effects of ICS therapy, and will elect not to take ICS even though these concerns are not valid. ${ }^{24-26}$ As an alternative, antileukotrienes can be used for mild asthma patients with key advantages including oral administration as well as a perceived lack of adverse effects. ${ }^{27}$ A 2003 Cochrane 
review, which contained 27 trials of asthma patients with mild to moderate asthma, analyzed the efficacy and safety of antileukotriene agents in comparison with ICS therapy. ${ }^{28}$ Eight trials specifically focused on mild asthma. Patients on an antileukotriene regimen were found to be $65 \%$ more likely to suffer an exacerbation requiring systemic steroids as well as a $160 \%$ increased risk of withdrawal due to poor asthma control when compared with patients on ICS. Improvements in lung function, symptom control, nocturnal awakenings, quality of life, symptom-free days and rescue medication usage were also found to significantly favor the utilization of ICS therapy.

As guidelines favor the minimum therapeutic dosage to maintain asthma control, a recent study analyzed the effect of reducing twice daily ICS therapy. ${ }^{29}$ Five hundred wellcontrolled patients with mild persistent asthma on twice daily fluticasone were randomly assigned to: 1) continue current regimen, 2) receive fluticasone and salmeterol once daily, or 3) montelukast once daily. Treatment failure occurred in approximately $30 \%$ of participants switched to montelukast in comparison with only $20 \%$ of patients who either continued fluticasone therapy or switched to combination therapy. However, across all treatment arms similar percentages of symptom-free days were observed. Ultimately, the authors concluded that while antileukotrienes are an option for mild persistent asthma, ICS therapy is still superior at maintaining asthma control. These sentiments are echoed in current guidelines. $^{3}$

\section{Immunotherapy}

Allergen-specific immunotherapy is stated to be a limited component of disease management in adults with asthma. ${ }^{3}$ Immunotherapy is indicated if asthma is triggered by exposure to airborne allergens, with poor response to asthma therapy or environmental controls, if avoiding the triggers is unrealistic or impossible. It is not indicated if the asthma is not of the allergic type. Controversy about its usage stems from its effectiveness to improve health outcomes conflicting with potential life-threatening adverse effects such as fatal anaphylaxis, as well as its cost and inconvenience because it requires coming in for regularly scheduled injections. A 2010 Cochrane review, with 88 trials including a total of 3792 participants (3459 having asthma) sought to assess the effectiveness of allergen-specific immunotherapy for asthma. ${ }^{30}$ The authors found a significant reduction in asthma symptom scores, medication requirements and allergenspecific bronchial hyper-reactivity as well as a nonsignificant reduction in nonspecific bronchial hyper-reactivity. No consistent effect on lung function was identified. The review identified concerns about the inability to clearly identify concealment allocation following randomization in numerous trials, along with significant patient heterogeneity being present across groups. The authors concluded that this review provides substantiation that allergen specific immunotherapy is efficacious in reducing asthma medication usage and asthma symptoms. However, from this review little guidance is available on the benefit of allergen-specific immunotherapy in comparison with other medications used in the treatment of asthma.

In another systematic review comprising 25 studies and 1706 patients, sublingual immunotherapy's (SLIT's) effectiveness was observed to provide modest benefit in patients predominantly suffering from mild to moderate asthma. ${ }^{31}$ An important clinical finding was that only mild adverse effects were observed, such as local pruritis, erythema, and edema, and no severe reactions were present. Consequently, SLIT may be considered as an alternative treatment to subcutaneous immunotherapy.

\section{Patient education}

Regardless of disease severity, patient education should be an integral component of asthma management as recommended in multiple guidelines. ${ }^{4,32}$ Key elements of education include basic information on the pathophysiology of asthma, a review of inhaler technique, as well as the need to adhere to treatment regimens. Moreover, it is important to address with openended questions potential patient concerns such as perceived side effects related to treatment. It is also important to develop and give to the patient a written action plan detailing the signs and symptoms suggestive that asthma is worsening, alongside the appropriate actions to take.

In 2002, a Cochrane review analyzed the impact of asthma self-management education programs alongside regular physician review on health outcomes. ${ }^{33}$ The review comprised 36 trials with approximately 6000 participants. The study showed that self-management education significantly reduced hospitalizations and emergency room visits. Furthermore, days off work or school, nocturnal asthma, and quality-of-life estimates were also shown to significantly improve. One concern that arose was the significant heterogeneity identified in unscheduled doctor visits, days off work, nocturnal symptoms, and quality-of-life outcomes, with possible causes being varying definitions between articles. In conclusion, the authors found that education improves health outcomes in asthmatic patients. Moreover, the effects of education programs can be 
increased with the incorporation of self-monitoring, regular medical review, and written action plans.

To further explore the effect of asthma education, a companion review was published to address the effects of education on health outcomes at its most superficial level, the exchange of knowledge concerning the causes of asthma, and its treatments. ${ }^{34}$ Limited asthma education excludes attitude or behavior modification as well as any attempt to develop self-management skills. Twelve trials were included in the systematic review. Results showed that limited asthma education did not reduce hospitalizations, doctor visits, time lost from work or school, and medication usage; nor did it improve lung function. However, in two studies, perceived asthma symptoms were shown to improve. Overall, these results support the theory that knowledge transfer alone has a limited capacity to modify behavior. Therefore, this limits its ability to modify health outcomes.

\section{Conclusion}

Mild asthma is still a considerable concern given that it comprises the largest number of asthma patients and because of the economic burden it places on health care systems. ICSs are the primary therapeutic option for mild persistent asthma, as well as mild intermittent asthma if the use of reliever medication is required more than twice weekly. This is due to the inability of $\mathrm{SABA}$ to provide effective current control as well as to prevent future severe asthma exacerbations. It is important to emphasize that at the low doses used in mild asthma, the systemic adverse effects are not a cause for concern. Multiple alternative treatment regimens have been assessed for mild disease. Intermittent ICS as well as antileukotrienes have both been considered; however, regular ICS therapy has been found to be more effective. The combination of ICSs and LABAs has been shown to be of no additional benefit to regular ICS therapy in patients with mild asthma. Alternative treatments such as chromoglycate, acupuncture, and phytotherapy can be considered; however, there is a lack of evidence supporting their utilization. Lastly, due to limitations and modest efficacy, allergen-specific immunotherapy plays a small role in asthma disease management.

Whether a patient suffers from either intermittent or persistent disease, all patients should be provided with a structured asthma education program. Patients should also receive a written action plan as it is important to inform patients not only on their current asthma control, but also on their potential risks in the future. A more in-depth analysis of the recommendation provided can be found in the Global Initiative for Asthma guidelines. ${ }^{5}$

\section{Disclosure}

Mr Shahidi reports no conflicts of interest in this work. Dr FitzGerald has been on the Advisory Boards for GSK, AstraZeneca, Novartis, Pfizer, Boehringer Ingelheim, Altana, Merck, and Topigen. He has also been on the Speakers Bureau for GSK, AstraZeneca, Boehringer Ingelheim, Pfizer, and Merck. Research funding was paid directly to UBC from CIHR, AZ, GSK, BI, Merck, Wyeth, Schering, Genentech, and Topigen. Dr FitzGerald is a member of the GINA Executive and Chair of the GINA Science Committee and is a member of the CTS Asthma Committee.

\section{References}

1. Sadatsafavi M, Lynd L, Marra C, et al. Direct health care costs associated with asthma in British Columbia. Can Respir J. 2010;17(2) 74-80.

2. Bateman ED, Hurd SS, Barnes PJ, et al. Global strategy for asthma management and prevention: GINA executive committee. Eur Resp J. 2008;31(1):143-178.

3. Robertson CF, Rubinfield AR, Bowes G. Pediatric asthma deaths in Victoria: the mild are at risk. Pediatr Pulmonol. 1992;13(2): 95-100

4. National Asthma Education and Prevention Program, National Heart, Lung and Blood Institute. Expert Panel Report 3: Guidelines for the Diagnosis and Management of Asthma. Bethseda, MD: NHLBI; 2007. NIH Publication No. 07-4051.

5. National Institutes of Health, Global Initiative For Asthma. GINA Report, Global Strategy for Asthma Management and Prevention. 2009 Available from: http://www.ginasthma.com/Guidelineitem. asp??11 $=2 \& 12=1 \&$ intId $=1561$. Accessed Oct 202010 .

6. Drazen JM, Isreal E, Boushey HA, et al. Comparison of regularly scheduled with as-needed use of albuterol in mild asthma. Asthma Clinical Research Network. N Engl J Med. 1996;335(12):841-847.

7. Vignola AM, Chanez P, Campbell AM, et al. Airway inflammation in mild intermittent and in persistent asthma. Am J Respir Crit Care Med. 1998;157(2):403-409.

8. Stankovic I, Pejcic T, Milenkovic B, et al. Is there any point in a corticosteroid treatment of intermittent asthma. Scientific WorldJournal. 2007; 7:1082-1108

9. Adams N, Bestall J, Jones PW. Budesonide versus placebo for chronic asthma in children and adults. Cochrane Database Syst Rev. 2001;4 CD003274.

10. Adams NP, Bestall JB, Malouf R, Lasserson TJ, Jones PW. Inhaled beclomethasone versus placebo for chronic asthma. Cochrane Database Syst Rev. 2005;1:CD002738.

11. Adams NP, Bestall JC, Lasserson TJ, Jones P, Cates CJ. Fluticasone versus placebo for chronic asthma in adults and children. Cochrane Database Syst Rev. 2008;4:CD003135.

12. Manning P, Gibson PG, Lasserson TJ. Ciclesonide versus placebo for chronic asthma in adults and children. Cochrane Database Syst Rev. 2008;2:CD006217.

13. Pauwels RA, Pederson S, Busse WW, et al. Early intervention with budesonide in mild persistent asthma: a randomised, double-blind trial. Lancet. 2003;361(9363):1071-1076.

14. Busse WW, Pederson S, Pauwels RA, et al. The inhaled steroid treatment as regular therapy in early asthma (START) study 5-year follow-up: effectiveness of early intervention with budesonide in mild persistent asthma. J Allergy Clin Immunol. 2008;121(5): 1167-1674.

15. Lipworth BJ. Systemic adverse effects of inhaled corticosteroid therapy: a systematic review and meta-analysis. Arch Intern Med. 1999;159(9): 941-955. 
16. Barnes PJ. Efficacy of inhaled corticosteroids in asthma.J Allergy Clin Immunol. 1998;102(4 Pt 1):531-538.

17. Boushey HA, Sorkness CA, King TS, et al. Daily versus as-needed corticosteroids for mild persistent asthma. N Engl J Med. 2005;352(15): $1519-1528$.

18. Bai TR, Vonk JM, Postma DS, et al. Severe exacerbations predict excess lung function decline in asthma. Eur Resp J. 2007;30(3):452.

19. British Thoracic Society. British Guideline on the Management of Asthma. Thorax. 2008;63(Suppl 4):iv1-121.

20. Stockl KM, Le L, Harada AS, et al. Use of controller medications in patients initiated on a long-acting B2-adrenergic agonist before and after safety alerts. Am J Health Syst Pharm. 2008;65(16): $1533-1538$.

21. Ni Chroinin M, Greenstone I, Lasserson TJ, et al. Addition of long-acting beta2-agonists to inhaled steroids as first line therapy for persistent asthma in steroid-naive adults and children. Cochrane Database Syst Rev. 2009;4:CD005307.

22. Papi A, Canonica GW, Maestrelli P, et al. Rescue use of beclomethasone and albuterol in a single inhaler for mild asthma. $N$ Engl $J$ Med. 2007;356(20):2040-2052.

23. Ducharme FM. Addition of anti-leukotriene agents to inhaled corticosteroids for chronic asthma. Cochrane Database Syst Rev. 2004;1: CD003133.

24. Bisgaard H, Nielson MD, Andersen B, et al. Adrenal function in children with bronchial asthma treated with beclomethasone diproprionate or budesonide. J Allergy Clin Immunol. 1988;81(6):1088-1095.

25. Kamada AK, Szefler SJ. Glucocorticoids and growth in asthmatic children. Pediatr Allergy Immunol. 1995;6(3):145-154.
26. Todd G, Dunlop K, McNaboe J, et al. Growth and adrenal suppression in asthmatic children treated with high-dose fluticasone proprionate. Lancet. 1996;348(9019):27-29.

27. Leff JA, Busse WW, Pearlman D, et al. Montelukast, a leukotrienereceptors antagonist, for the treatment of mild asthma and exerciseinduced bronchoconstriction. N Engl J Med. 1998;339(3):147-152.

28. Ducharme F, di Salvio F. Anti-leukotriene agents compared to inhaled corticosteroids in the management of recurrent and/or chronic asthma in adults and children. Cochrane Database Syst Rev. 2004;1: CD002314.

29. Peters SP, Anthonisen N, Castro M, et al. Randomized comparison of strategies for reducing treatment in mild persistent asthma. $N$ Engl J Med. 2007;356(20):2027-2039.

30. Abramson MJ, Puy RM, Weiner JM. Injection allergen immunotherapy for asthma. Cochrane Database Syst Rev. 2010;8:CD001186.

31. Calamita Z, Saconato H, Pela AB, et al. Efficacy of sublingual immunotherapy in asthma; systematic review of randomized-clinical trials using the Cochrane Collaboration method. Allergy. 2006;61(10): 1162-1172.

32. Bailey WC, Clark NM, Gotsch AR, et al. Asthma prevention. Task Force on Research and Education for the Prevention and Control of Respiratory Diseases. Chest. 1992;102 Suppl 3:216S-231S.

33. Gibson PG, Powell H, Wilson A, et al. Self-management education and regular practitioner review for adults with asthma. Cochrane Database Syst Rev. 2002;3:CD001117.

34. Gibson PG, Powell H, Wilson A, et al. Limited (information only) patient education programs for adults with asthma. Cochrane Database Syst Rev. 2002;1:CD001005.

\section{Publish your work in this journal}

The Journal of Asthma and Allergy is an international, peer-reviewed open-access journal publishing original research, reports, editorials and commentaries on the following topics: Asthma; Pulmonary physiology; Asthma related clinical health; Clinical immunology and the immunological basis of disease; Pharmacological interventions and

\section{Dovepress}

new therapies. Issues of patient safety and quality of care will also be considered. The manuscript management system is completely online and includes a very quick and fair peer-review system, which is all easy to use. Visit http://www.dovepress.com/testimonials.php to read real quotes from published authors. 